\title{
Validity of the One-Dimensional Limp Model for Porous Media
}

\author{
O. Doutres, N. Dauchez, J.-M. Genevaux, O. Dazel
}

\begin{abstract}
A straightforward criterion for determining the validity ofthe limp model validity for porous materials is addressed here. The limp model is an "equivalent fluid" model which gives a better description of porous behavior than the well known "rigid frame" model. It is derived from the poroelastic Biot model, assuming that the frame has no bulk stiffness. A criterion is proposed for identifying the porous materials for which the limp model can be used. It relies on a new parameter, the Frame Stiffness Influence FSI, based on porous material properties. The critical values of FSI under which the limp model can be used are determined using $1 D$ analytical modeling for a specific boundary set: radiation of a vibrating plate covered by a porous layer.
\end{abstract}

Keywords: Porous media, fibrous, limp model, Biot model, criterion.

\section{Introduction}

In recent years, poroelastic numerical models using finite element method have been widely developed to improve the acoustic efficiency of porous materials used in the aeronautic and automotive industries. Classical methods use the Biot theory $[1,2]$ to account for the displacements of both solid and fluid phases. To model three dimensional applications, six or four degrees-of-freedom per node are required, depending on the chosen formulation [5, 6].These numerical methods enable us to predict the structural and fluid coupling induced by the poroelastic medium without any kinematic or geometrical assumptions. However, for large size finite element models, these methods can require significant computational time.

To overcome this limitation, we can consider that the porous layer behaves like a dissipative fluid. Two porous one-wave formulations can be found: (i) the rigid frame model assumes that the solid phase remains motionless [2], and (ii) the limp model assumes that the stiffness of the solid phase is zero but takes into account its inertial effects [8, 9, 10, 11, 12]. Because the motion of the solid phase is considered in the limp model, this model has to be preferred for most of applications, as e.g., in means of transport (cars, trains, aircrafts), where the porous layers are bonded to vibrating plates. However, the model is valid since the frame flexibility of the porous material has little influence on the vibroacoustic response of the system.

In a preceding paper [11], a criterion was proposed for identifying the porous materials and the frequency bands for which the limp model can be used according to the boundary conditions applied to the layer. The identification process is based on a parameter, the Frame Stiffness Influence (FSI), determined from the properties of the porous material. This parameter, developed from the Biot theory $[1,2]$ quantifies the intrinsic influence of the solid-borne wave [2] on the displacement of the interstitial fluid and is frequency dependent. In this study, the parameter FSI was compared to critical values obtained for different boundary conditions and porous thicknesses to give an estimation of the frequency bands for which the limp model can be used.

In this paper, the identification process is more straightforward to give a first estimation on the accuracy of using the limp model in the whole frequency range. It is based on a frequency independent parameter FSIr derived from FSI. Critical values of FSIr above which the limp model cannot be used are determined for porous materials from 1 to $5 \mathrm{~cm}$ in thickness and for a specific boundary condition set (see Fig. 3). Here we present the sound radiation of a porous layer backed by a vibrating wall.

\section{Porous material modeling}

\subsection{Biot theory}

According to Biot theory, three waves propagate in a porous media: two compressional waves and a shear wave. In this work, the applications are one-dimensional and only the two compressional waves are considered. The motion of the poroelastic medium is described by the macroscopic displacement of the solid and fluid phase, respectively denoted $u^{\mathrm{s}}$ and $u$. Assuming a harmonic time dependence, the equation of motion can be written in the following form [11]:

$$
\begin{aligned}
& -\omega^{2} \frac{\widetilde{\rho}_{12}}{\phi} \widetilde{\Gamma} u^{\mathrm{s}}-\omega^{2} \frac{\widetilde{\rho}_{22}}{\phi} \widetilde{\gamma} u^{\mathrm{f}}=\hat{P} \nabla^{2} u^{\mathrm{s}}, \\
& -\omega^{2} \widetilde{\rho}_{12} u^{\mathrm{s}}-\omega^{2} \widetilde{\rho}_{22} u^{\mathrm{f}}=\widetilde{Q} \nabla^{2} u^{\mathrm{s}}+\widetilde{R} \nabla^{2} u^{\mathrm{f}},
\end{aligned}
$$

with

$$
\widetilde{\Gamma}=\phi\left(\frac{\widetilde{\rho}_{11}}{\widetilde{\rho}_{12}}-\frac{\widetilde{Q}}{\widetilde{R}}\right), \widetilde{\gamma}=\phi\left(\frac{\widetilde{\rho}_{12}}{\widetilde{\rho}_{22}}-\frac{\widetilde{Q}}{\widetilde{R}}\right) .
$$

The tilde symbol indicates that the associated physical property is complex and frequency-dependent. The inertial coefficients $\widetilde{\rho}_{11}$ and $\widetilde{\rho}_{22}$ are the modified Biot density of the solid and fluid phase, respectively. The inertial coefficient $\widetilde{\rho}_{12}$ accounts for the interaction between the inertial forces of the solid and fluid phases together with the viscous dissipation. In Eq. $(1,2), \hat{P}$ is the bulk modulus of the frame in vacuum

$$
\hat{P}=\frac{E(1+j \eta)(1-v)}{(1-2 v)(1+v)},
$$

with $E$ the Young modulus, $\eta$ the loss factor, $v$ the Poisson ratio of the frame, $\widetilde{R}$ is the bulk modulus of the fluid phase, $\widetilde{Q}$ quantifies the potential coupling between the two phases, and $\phi$ is the porosity.

In the geometry considered here, the displacement of each phase is due to the propagation of two compressional 
waves traveling in both directions. They can be written in the form

$$
\begin{aligned}
& u^{\mathrm{s}}(x)=X_{1}+X_{2}, \\
& u^{\mathrm{f}}(x)=\mu_{1} X_{1}+\mu_{2} X_{2},
\end{aligned}
$$

where $X_{i}=S_{i} \cos \left(\delta_{i} x\right)+D_{i} \sin \left(\delta_{i} x\right)$ is the contribution of each compressional wave $i=1,2, S_{i}$ and $D_{i}$ is set by the boundary conditions. These waves are characterized by a complex wave number $\delta_{i}(i=1,2)$ and a displacement ratio $\mu_{i}$. This ratio indicates in which medium the waves mainly propagate. Here, the wave with the subscript $i=1$ propagates mainly in the fluid phase and is referred to as the airborne wave. The wave with the subscript $i=2$ propagates mainly in the solid phase and is referred to as the frame-borne wave.

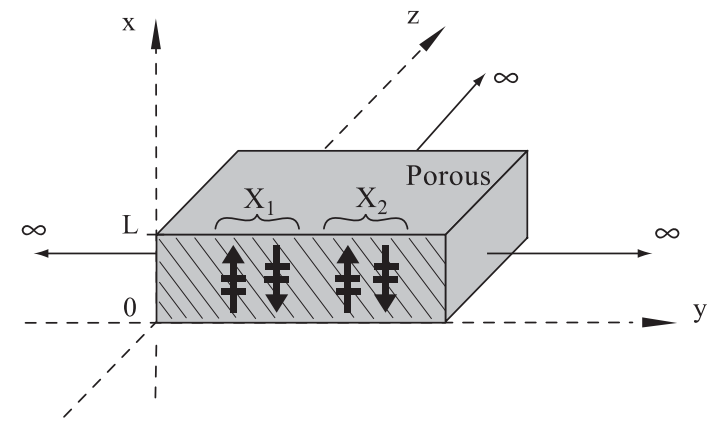

Fig. 1: One-dimensional porous modeling

\subsection{Limp assumption}

The limp model is derived from the Biot theory. It is based on the assumption that the frame has no bulk stiffness $[8,9$, $10,11,12]: \stackrel{P}{P}=0$. It is likely associated to soft materials like cotton and glass wool. This model describes the propagation of one compressional wave in a medium that has the bulk modulus of the air in the pores and the density of the air modified by the inertia effect of the solid phase and its interaction with the fluid phase.

Hence, by considering the assumption $\hat{P}=0$ in Eq. (1), we get a simple relation between the displacements of both solid and fluid phases. Then, substituting the solid displacement in Eq. (2) gives the propagation equation on $u^{\mathrm{f}}$

$$
\widetilde{K}_{\mathrm{f}} \nabla^{2} u^{\mathrm{f}}+\omega^{2} \widetilde{\rho}_{\lim p} u^{\mathrm{f}}=0,
$$

with $\widetilde{K}_{\mathrm{f}}$ the bulk modulus of the air in the pores and $\widetilde{\rho}_{\lim p}$ the modified density of the air. An expression of these coefficients can be found in reference $[11,12]$.

\section{Influence of frame stiffness}

The aim of this section is to propose a parameter based on the properties of the porous material which quantifies the influence of the frame stiffness on the porous behavior. This parameter is called FSI for Frame Stiffness Influence.

\subsection{Development of the frequency dependent parameter FSI}

The limp model can be used when the contribution of the frame-borne wave is negligible in the considered application.
This approximation implies in the expressions of the solid and fluid displacements (Eq. $(5,6))$ that:

- the contribution of the airborne wave $X_{1}$ is great compared to the contribution of the frame-borne wave $X_{2}$; this condition depends mainly on the boundary conditions : one configuration will be presented in section 4 to set critical values of the FSI parameter,

- considering the fluid motion (Eq. (6)), the displacement ratio $\mu_{1}$ associated to the airborne wave is great compared to the displacement ratio $\mu_{2}$ associated to the frame-borne wave: $\mu_{2} / \mu_{1}<<1$; this condition is independent from the boundary conditions and will be used to build the FSI parameter.

Hence, the FSI parameter is based on the assumption of the limp model can be used when the frame-borne wave contribution is negligible in the considered application. The associated condition, $\mu_{2} / \mu_{1}<<1$, can be written in terms of a frequency dependent parameter, FSI, expressed as a ratio of two characteristic wave numbers [11]

$$
\mathrm{FSI}=\frac{\delta_{\lim p}^{2}}{\delta_{c}^{2}}=\frac{\widetilde{\rho}_{\lim p}}{\widetilde{\rho}_{c}} \frac{\hat{P}}{\widetilde{K}_{\mathrm{f}}} .
$$

$\delta_{\lim p}=\omega \sqrt{\widetilde{\rho}_{\lim p} / \widetilde{K}_{\mathrm{f}}}$ is the wave number derived from the limp model, and $\delta_{c}=\omega \sqrt{\widetilde{\rho}_{c} / \hat{P}}$ is the wave number of a wave, called the $c$ wave, that propagates in a medium that has the bulk modulus of the frame in a vacuum and the density of the frame in a fluid

$$
\widetilde{\rho}_{c}=\rho_{1}-\frac{\widetilde{\rho}_{12}}{\phi},
$$

with $\rho_{1}$ the mass density of the porous material.

Table 1: Measured properties of materials B and C

\begin{tabular}{|l|c|c|}
\hline Porous & B & C \\
\hline Air flow resistivity: $\sigma\left(\mathrm{kNs} / \mathrm{m}^{4}\right)$ & 23 & 57 \\
Porosity: $\phi$ & 0.95 & 0.97 \\
Tortuosity: $\alpha_{\infty}$ & 1 & 1.54 \\
Viscous lenght: $\Lambda(\mu \mathrm{m})$ & 54.1 & 24.6 \\
Thermal lenght: $\Lambda(\mu \mathrm{m})$ & 162.3 & 73.8 \\
\hline Frame density: $\rho_{1}\left(\mathrm{~kg} / \mathrm{m}^{3}\right)$ & 58 & 46 \\
Young's modulus at $\mathbf{5} \mathbf{H z}: E(\mathrm{kPa})$ & 17 & 214 \\
Structural loss factor at 5Hz: $\boldsymbol{\eta}$ & 0.1 & 0.115 \\
Poison's ratio: $\boldsymbol{v}$ & 0 & 0.3 \\
\hline
\end{tabular}

Fig. 2 presents the FSI for the two characteristic materials $\mathrm{B}$ and $\mathrm{C}$ [ 11 ]. Material $\mathrm{B}$ is a high-density fibrous material and material $\mathrm{C}$ is a polymer foam with a stiff skeleton and high airflow resistivity. The properties of these materials presented in Table 1 were measured in our laboratory. 


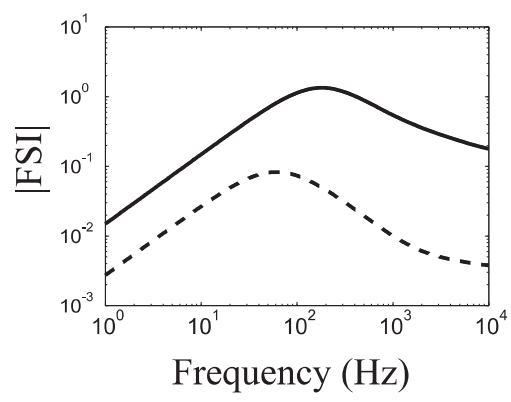

Fig. 2: FSI of material (- -) B and (-) C

This figure shows that the FSI parameter has a bell shape in which amplitude increases with the bulk modulus of the porous skeleton. The maximum amplitude occurs at the decoupling frequency defined by Zwikker and Kosten [13]:

$$
f_{\mathrm{ZK}}=\frac{\phi^{2} \sigma}{2 \pi \rho_{1}} \text {. }
$$

This frequency indicates the frequency below which the viscous forces on the material are superior to the inertial forces per unit volume. It is generally used to determine the critical frequency above which an acoustical wave propagating in the fluid phase would not exert a sufficient force to generate vibrations in the solid phase.

\subsection{A simplified frequency independent parameter $\mathrm{FSI}_{r}$}

The main objective of the paper is to propose a straightforward identification process which is more easy to carried out compared to the one presented in ref [11]. The criterion proposed in this paper consists in comparing a frequency independent parameter which characterizes the frame influence with critical value. This frequency independent parameter is set as the maximum value of FSI to ensure the uniqueness of the solution in the whole frequency range. Thus, as mentioned previously, it can be approached from the mass densities of both the limp waves and the $c$ waves expressed at the frequency.

Assuming that the density of air $\rho_{\mathrm{f}}$ is negligible compared with that of the porous material $\rho_{1}$, these densities are given by

$$
\begin{aligned}
& \widetilde{\rho}_{c}\left(f_{Z K}\right) \approx \rho_{1}\left(1-j \frac{1}{\phi}\right), \\
& \widetilde{\rho}_{\lim p}\left(f_{Z K}\right) \approx \rho_{1} \frac{(1-j \phi)}{\left(1+\phi^{2}\right)} .
\end{aligned}
$$

Hence, the modulus of the maximum FSI at $f_{\mathrm{ZK}}$ is given by

$$
\mathrm{FSI}_{r}=\left|\operatorname{FSI}\left(f_{\mathrm{ZK}}\right)\right| \approx \frac{\hat{P}}{P_{0}} \frac{\phi}{1+\phi^{2}} .
$$

$\mathrm{FSI}_{r}$ is then easy to calculate and requires measurement of the bulk modulus of the skeleton $\hat{P}$ and the porosity $(\phi)$. The two parameters $\mathrm{FSI}_{r}$ and $f_{\mathrm{ZK}}$ are given in Table 2 for materials $\mathrm{B}$ and $\mathrm{C}$.
Table 2: Simplified FSI parameter of materials B and C

\begin{tabular}{|l|c|c|}
\hline Material & B & G \\
\hline$f_{\mathrm{ZK}}(\mathbf{H z})$ & 57 & 186 \\
\hline $\mathrm{FSI}_{r}$ at $f_{\mathrm{ZK}}$ & $8.4210^{-2}$ & 1.43 \\
\hline
\end{tabular}

\section{Determination of critical FSI values}

The previous section introduced the simple parameter FSIr based on the physical properties of the material. The next step is to identify, for a specific boundary condition set, the critical values of FSI under which the limp model can be used instead of the Biot model. These critical values are determined from the difference between the limp and the Biot model carried out for a wide range of acoustic materials: hence, the critical FSI value is independent of the tested material.

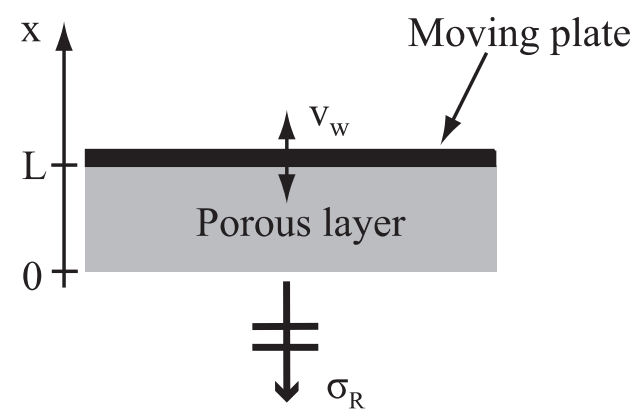

Fig. 3: Sound radiation of a porous layer backed by a vibrating wall

The chosen configuration is presented in Fig. 3. The porous layer is excited by a vibrating plate at $x=L$ and radiates in an infinite half-space at $x=0$. This configuration corresponds to trim panels, cars roofs or airplane floors. The radiation efficiency factor $\sigma_{\mathrm{R}}$, defined as the ratio of the acoustic power radiated $\Pi_{a}$ over the vibratory power of the piston $\Pi_{v}$, is used as a vibroacoustic response:

$$
\sigma_{\mathrm{R}}=\frac{\Pi_{a}}{\Pi_{v}}=\frac{p(0) v^{*}(0)}{\rho_{f} c_{f} v_{\mathrm{W}}^{2}}
$$

A vibrating surface area of $1 \mathrm{~m}^{2}$ is considered here. Boundary conditions associated to this configuration are [14]: continuity of stress and total flow at $x=0$. At $x=L$, the velocity of the fluid and the velocity of the frame are both equal to the wall velocity

$$
j \omega u^{\mathrm{s}}(L)=j \omega u^{\mathrm{f}}(L)=v_{\mathrm{w}}
$$

The vibroacoustic response is derived using the Transfer Matrix Method (TMM) [2]. This method assumes that the multilayer has infinite lateral dimensions and uses a representation of plane wave propagation in different media in terms of transfer matrices. To ensure a one-dimensional representation, the multilayer is excited by plane waves with normal incidence. The porous is either simulated using layer the Biot model or the limp model presented in section 2. Fig. 4 shows the Biot and limp simulations the radiation efficiency of materials B and C $2 \mathrm{~cm}$ in thickness. For 

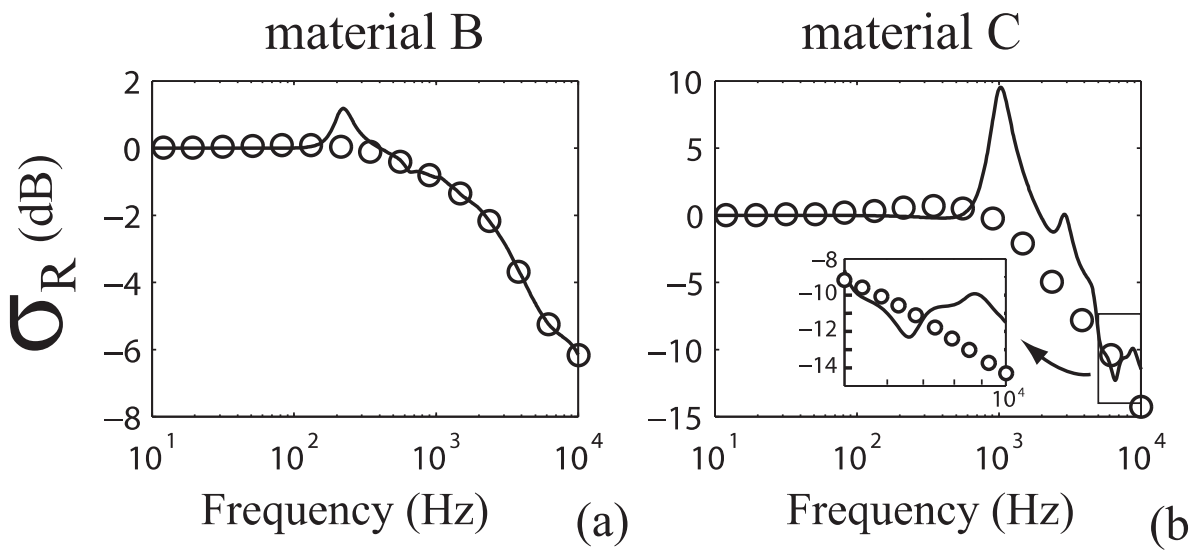

Fig. 4: Radiation efficiency simulated with the Biot model (solid line) and the limp model (circles): (a) material B, (b) material C

both materials, an increase of the radiation efficiency is observed around the first $\lambda / 4$ resonance frequency of the frame: around $200 \mathrm{~Hz}$ for material $\mathrm{B}$ and $1000 \mathrm{~Hz}$ for material C.

To determine the critical FSI value, the difference between the two models is derived by the absolute value of the difference of the two responses $\Delta \sigma_{\mathrm{R}}=\left|\sigma_{\mathrm{R}(\text { Biot })}\right|-\left|\sigma_{\mathrm{R}(\lim p)}\right|$. The maximum accepted difference between the two models is set to $3 \mathrm{~dB}$, and corresponds to a classical industrial demand. In order to determine a critical FSI value independent of the tested material, the difference between the two simulations is plotted as a function of the frequency dependent parameter FSI for a wide variety of porous materials (256 simulated materials). The critical FSI value corresponds to the minimum FSI value for which the model difference exceeds the maximum acceptable value of $3 \mathrm{~dB}$ [11].

The abacus given in Fig. 5 presents the minimum FSI critical values determined for 5 different porous thicknesses. For a given material, the limp model can be used if its $\mathrm{FSI}_{r}$ is situated below the critical value (white area of the abacus), and the Biot model should be preferred if FSIr exceeds the critical value (gray area of the abacus).

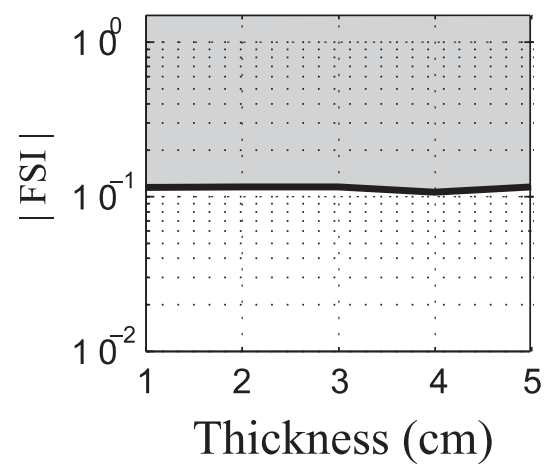

Fig. 5: Evolution of FSI critical value as a function of the porous thickness

\section{Discussion and conclusion}

A straightforward method is proposed to determine if the limp model can be used in the whole frequency range $(1-10000 \mathrm{~Hz})$. The procedure is as follows:

- Two properties of the porous materials, $\hat{P}$ and, $\phi$ have to be measured. (see Table 1 for materials B and C).
- The parameter $\mathrm{FSI}_{r}$ is evaluated using Eq. (13).

- The critical values of FSI are chosen in Fig. 5 according to the thickness of the porous layer.

- $\mathrm{FSI}_{r}$ is finally compared to the critical values: the limp model can be used in the whole frequency range if $\mathrm{FSI}_{r}$ is below the FSI critical value.

In the case of material C, $\mathrm{FSI}_{r}$ is equal to 1.4 (see Table 2), which is above the FSI critical values of the radiation configuration and for all thicknesses: the Biot model should be preferred for all layer thicknesses. The $\mathrm{FSI}_{r}$ of material $\mathrm{B}$ is equal to $8.410^{-2}$ (see Table 2 ), which is below the FSI critical values of the radiation configuration for all thicknesses: the limp model can be used for all porous thicknesses. These predictions agree with the simulations presented in Fig. 4. Note that for material B, the increase the radiation efficiency induced by the frame motion do not exceed the maximum accepted difference between the Biot and limp models of $3 \mathrm{~dB}$.

The proposed method is easy to carry out and allows to estimate if the one-dimensional limp model can be used instead of the complete Biot model without making any numerical simulations of the configuration nor experimental studies. Note that the use of the limp model can be particularly interesting in order to decrease the computational time for large finite element calculations which include porous materials. The criterion method has been presented here in the case of the radiation efficiency of a plate covered by a porous layer of different thicknesses. It has been shown that the prediction of the material for which the limp model can be used is in close agreement with $1 \mathrm{D}$ simulations.

\section{Acknowledgments}

This study was supported in the framework of the CREDO research project co-funded by the European Commission.

\section{References}

[1] Biot, M. A.: The Theory of Propagation of Elastic Waves in a Fluid-Saturated Porous Solid I. Low Frequency Range; II Higher Frequency Range. J. Acoust. Soc. Am. Vol. 28 (1956), p. 168-191. 
[2] Allard, J. F.: Propagation of Sound in Porous Media: Modelling Sound Absorbing Materials. London: Elsevier Applied Science, 1993.

[3] Panneton, R., Atalla, N.: An Efficient Scheme for Solving the Three-Dimensional Elasticity Problem in Acoustics. J. Acoust. Soc. Am. Vol. 101(1998), No. 6, p. 3287-3298.

[4] Hörlin, N. E., Nordström, M., Göransson P.: A 3-D Hierarchical FE Formulation of Biot's Equations for Elastoacoustic Modeling of Porous Media.J. Sound. Vib. Vol. 254 (2001), No. 4, p. 633-652.

[5] Atalla, N., Panneton, R., Debergue, P.: A Mixed Displacement-Pressure Formulation for Poroelastic Materials. Journal Acoust. Soc. Am. Vol. 104 (1998), No. 4, p. 1444-1452, .

[6] Dauchez, N., Sahraoui, S., Atalla, N.: Convergence of Poroelastic Finite Elements Based on Biot Displacement Formulation. J. Acoust. Soc. Am. Vol. 109 (2001), No. 1, p. 33-40.

[7] Rigobert, S., Atalla, N., Sgard, F. : Investigation of the Convergence of the Mixed Displacement Pressure Formulation for Three-Dimensional Poroelastic Materials Using Hierarchical Elements. J. Acoust. Soc. Am. Vol. 114 (2003), No. 5, p. 2607-2617.

[8] Beranek, L. L.: Acoustical Properties of Homogeneous, Isotropic Rigid Tiles and Flexible Blankets. J. Acoust. Soc. $A m$. Vol. 19 (1947), No. 4, p. 556-568.

[9] Ingard, K. U.: Notes on Sound Absorption Technology, Noise Control Foundation. New York, 1994.
[10] Dazel, O., Brouard, B., Depollier, C., Griffiths, S.: An Alternative Biot's Displacement Formulation for Porous Materials. J. Acoust. Soc. Am. Vol. 121 (2007), No. 6, p. 3509-3516.

[11] Doutres, O., Dauchez, N., Genevaux, J. M., Dazel, O.: Validity of the Limp Model for Porous Materials: A Criterion Based on Biot Theory.J. Acoust. Soc. Am. Vol. 122 (2007), No. 4, p. 2038-2048.

[12] Panneton, R.: Comments on the Limp Frame Equivalent Fluid Model for Porous Media. J. Acoust. Soc. Am. Vol. 122(2007), No. 6, p. EL 217-222.

[13] Zwikker, C., Kosten, C. W.: Sound Absorption Materials. New York: Elsevier Applied Science, 1949.

[14] Doutres, O., Dauchez, N., Genevaux, J. M.: Porous Layer Impedance Applied to a Moving Wall: Application to the Radiation of a Covered Piston. J. Acoust. Soc. Am. Vol. 121 (2007), p. 206-213.

\section{Olivier Doutres}

Nicolas Dauchez

e-mail: nicolas.dauchez@univ-lemans.fr

Jean-Michel Genevaux

Olivier Dazel

LAUM, CNRS, Université du Maine

Av. O. Messiaen

72095 Le Mans, France 